\title{
Finite-gain $\mathcal{L}_{\infty}$ stability from disturbance to output of impulsive systems with time delay
}

\author{
Ping Li ${ }^{\mathrm{a}, \mathrm{b}}$, Xinzhi Liu ${ }^{\mathrm{b}}$, Wu Zhao ${ }^{\mathrm{c}, *}$ \\ a School of Computer Science and Technology, Southwest Minzu University, Chengdu, 610041, P. R. China. \\ ${ }^{b}$ Department of Applied Mathematics, University of Waterloo, Waterloo, Ontario, Canada N2L 3G1. \\ ${ }^{c}$ School of Management and Economics, University of Electronic Science and Technology of China, Chengdu, 610054, P. R. China. \\ Communicated by R. Saadati
}

\begin{abstract}
This paper studies finite-gain $\mathcal{L}_{\infty}$ stability from disturbance to output of delayed impulsive systems. By employing the method of Lyapunov function, several criteria of finite-gain $\mathcal{L}_{\infty}$ stability from disturbance to output are established. It shows that the linear delayed differential systems can be finite-gain $\mathcal{L}_{\infty}$ stabilized from disturbance to output using impulsive feedback control even there is unstable matrix. Moreover, delayed differential equations also may be finite-gain $\mathcal{L}_{\infty}$ stable from disturbance to output under an appropriate sequence of impulses treated as disturbances. Two examples and their simulations are also given to illustrate our results. (c)2017 All rights reserved.
\end{abstract}

Keywords: Impulsive control, impulsive disturbance, Lyapunov function, finite-gain $\mathcal{L}_{\infty}$ stability. 2010 MSC: 34A37, 34C11.

\section{Introduction}

Issues of input-output stability and controller design have been under investigation in the last decades $[1,3,4,7,8,10,12-14,16,17]$. The strength of input-output stability theory is that it provides a method for anticipating the qualitative behavior of a feedback system with only rough information about the feedback components [14]. Disturbance considered as one kind of exogenous inputs is frequently a source of generation of oscillation and instability and poor performance and commonly exists in various mechanical, biological, physical, chemical engineering, economic systems. In this paper, we will study disturbance-output property of delayed systems via impulsive control based on the results of input-output stability analysis.

On the other hand, many physical systems such as the orbital transfer of satellite, ecosystems management and control of money supply in a financial market are modeled in the impulsive differential systems. Significant progress has been made in the control theory of impulsive delay differential systems

\footnotetext{
*Corresponding author

Email addresses: liping925@126.com (Ping Li), xinzhi.liu@uwaterloo.ca (Xinzhi Liu), zhaowu@uestc.edu.cn (Wu Zhao)
} doi:10.22436/jnsa.010.04.25 
in the recent years, see $[2,5,9,11]$ and references therein. However, there is little effort on the study of disturbance-output property for delayed impulsive systems by Lyapunov method.

Motivated by the aforementioned discussions, we will investigate bounded behaviors of systems for every bounded disturbance by impulsive control. Impulsive feedback control is designed to guarantee the output of delayed systems will remain bounded for any bounded disturbance and several criteria of finite-gain $\mathcal{L}_{\infty}$ stability from disturbance to output for delayed impulsive system are established using the method of Lyapunov function. Our results show that no matter whether the delay-free systems are unstable or stable, impulsive differential systems are to be bounded for any bounded disturbance. The rest of this paper is organized as follows. In Section 2, we formulate the problem of impulsive control and introduce some definitions and a lemma. We then establish, in Section 3, several criteria of finite-gain $\mathcal{L}_{\infty}$ stability from disturbance to output for impulsive differential systems. These criteria may be used for impulse feedback control designs. Finally, some examples are discussed to illustrate our results.

\section{Problem formulation}

Consider the continuous-time system

$$
\left\{\begin{array}{l}
\dot{x}(t)=A x(t)+B x(t-\tau)+u(t)+w(t), \\
y(t)=C x(t), \\
x_{t_{0}}(s)=x\left(t_{0}+s\right)=\varphi(s), \quad s \in[-\tau, 0],
\end{array}\right.
$$

where $x(t), u(t), y(t), w(t) \in R^{n}$ are the state vector, control input, control output, disturbance of the systems, respectively; $\tau>0$ is known constant time delay; $\phi(t) \in C\left([-\tau, 0], R^{n}\right)$ is an initial function. To obtain the bounded output of systems, we let

$$
u(t)=F x(t),
$$

where $F$ is the feedback gain matrix. The output of systems (2.1) can be guaranteed bounded for any bounded disturbance by the design of feedback gain matrix. As is mentioned above, impulsive control has wide applications. In this circumstances, some natural questions arise: Can bounded disturbances produce bounded responses (outputs) by impulsive control? What are the conditions ensuring that the boundedness properties of (2.1) still hold, when there exists an impulsive disturbance with the effect of sudden change of the state of the systems? Do the systems have the property of robustness for the impulsive disturbances?

In order to investigate the impulsive effect on boundedness of output, we let

$$
u(t)=\sum_{k=1}^{\infty} F x(t) \delta\left(t-t_{k}^{-}\right),
$$

where $\delta(t)$ is Dirac delta-function defined by

$$
\left\{\begin{array}{l}
\delta(t)=0, \quad t \neq 0 \\
\int_{-\infty}^{+\infty} \delta(t) d t=1
\end{array}\right.
$$

Then systems (2.1) with (2.2) become

$$
\begin{cases}\dot{x}(t)=A x(t)+B x(t-\tau)+w(t), & t \neq t_{k}, \\ \Delta x\left(t_{k}\right)=F_{k} x\left(t_{k}^{-}\right), & t=t_{k}, \\ y(t)=C x(t), & t \geqslant t_{0}, \\ x_{t_{0}}(s)=x\left(t_{0}+s\right)=\varphi(s), & s \in[-\tau, 0],\end{cases}
$$

where $0 \leqslant t_{0}<t_{1}<t_{2}<\cdots<t_{k}<\cdots$, with $t_{k} \rightarrow \infty$ as $k \rightarrow \infty ; \Delta x(t)=x(t)-x\left(t^{-}\right)$; and $x_{t}, x_{t^{-}} \in P C\left([-\tau, 0], R^{n}\right)$ are defined by $x_{t}(s)=x(t+s), x_{t^{-}}(s)=x\left(t^{-}+s\right)$ for $-\tau \leqslant s \leqslant 0$, respectively. Next, we shall discuss the finite-gain $\mathcal{L}_{\infty}$ stability from disturbance to output of systems (2.3).

Let us begin with establishing some definitions and a lemma which will be used in the proof of the theorems. 
Definition 2.1. A real-valued vector $w(t) \in \mathcal{L}_{\infty}^{n}$, if $\|w\|_{\mathcal{L}_{\infty}}=\sup _{\mathrm{t}_{0} \leqslant \mathrm{t}<\infty}\|w(t)\|<+\infty$.

Definition 2.2. The control systems (2.3) are said to be finite-gain $\mathcal{L}_{\infty}$ stable from disturbance (here $w$ ) to output (here $y$ ), if there exist nonnegative constants $\gamma$ and $\theta$ such that

$$
\|y(t)\| \leqslant \gamma\|w\|_{\mathcal{L}_{\infty}}+\theta,
$$

for all $w(t) \in \mathcal{L}_{\infty}^{n}, t \geqslant t_{0}$.

Remark 2.3. Definition 2.2 relates the output of the systems directly to the disturbance, namely, if the systems are finite-gain $\mathcal{L}_{\infty}$ stable from $w$ to $y$, then for every bounded disturbance $w(t)$, the output $y(t)$ is bounded. It is defined according to the [6, Definition 5.1], a concept of stability in the input-output sense. The constant $\theta$ in Definition 2.2 is called the bias term.

Remark 2.4. The norm function captures the "size" of signals. The $\infty$-norm is useful when amplitude constraints are imposed on a problem, and the 2-norm is of more help in the context of energy constraints. We will typically be interested in measuring signals of the $\infty$-norm.

Lemma 2.5 ([15]). If $u: R_{+} \rightarrow R$ is a continuous solution of

$$
x(t) \leqslant f(t)+\int_{0}^{t} k(s) x(s) d s, \quad t \in R_{+},
$$

(where $f: R_{+} \rightarrow R$ and $k: R_{+} \rightarrow R_{+}$, are continuous functions) then

$$
u(t) \leqslant f(t)+\int_{0}^{t} k(s) \exp \left(\int_{0}^{t} k(r) d r\right) f(s) d s, \quad t \in R_{+} .
$$

\section{Stabilization criteria}

In this section, the finite-gain $\mathcal{L}_{\infty}$ stability from disturbance to output of impulsive systems (2.3) is discussed. Theorem 3.1 shows that by impulsive control systems (2.3) can be finite-gain $\mathcal{L}_{\infty}$ stabilized from $w$ to $y$ even, if the delay-free systems are unstable. When the delay-free systems are stable, we can get the same results showed in Theorem 3.3 where impulses are treated as disturbances.

Theorem 3.1. Assume that there exist a positive definite matrix $P$ and constants $\alpha_{i} \in R^{+}, i=1,2,3, d_{k} \in$ $\mathrm{R}^{+}, \mathrm{k} \in \mathrm{N}$, and $\beta$, such that

$$
\begin{aligned}
& {\left[\begin{array}{ccc}
A^{\top} P+P A-\alpha_{1} P & P B & P \\
* & -\alpha_{2} P & 0 \\
* & * & -\alpha_{3} I
\end{array}\right] \leqslant 0,} \\
& \left(I+F_{k}\right)^{\top} P\left(I+F_{k}\right)-d_{k} P \leqslant 0 \text { for each } k=1,2, \cdots,
\end{aligned}
$$

and

$$
\tau \leqslant t_{k}-t_{k-1} \leqslant \beta \leqslant \frac{\ln \alpha_{1}\left(\alpha_{1}+b\right)(a-c)-\ln \alpha_{2}-\ln \left(a \alpha_{1}+a b-b c\right)-\ln \left(1-e^{-\alpha_{1} \tau}\right)}{\alpha_{1}+b}
$$

and

$$
0<d_{k} \leqslant \frac{(a-c)-\frac{\alpha_{2}}{\alpha_{1}}\left(a-c+\frac{\alpha_{1} c}{\alpha_{1}+b}\right) e^{\left(\alpha_{1}+b\right) \beta}\left(1-e^{-\alpha_{1} \tau}\right)}{\left(a-c+\frac{\alpha_{1} c}{\alpha_{1}+b}\right) e^{\left(\alpha_{1}+b\right)\left(t_{k}-t_{k-1}\right)}}<1,
$$

where

$$
a=\lambda_{\max }(P)\|\phi\|_{\tau}^{2}\left(1+\frac{\alpha_{2}}{\alpha_{1}}-\frac{\alpha_{2}}{\alpha_{1}} e^{-\alpha_{1} \tau}\right)+\frac{\alpha_{3}}{\alpha_{1}}\|w\|_{\mathcal{L}_{\infty}}^{2}, \mathrm{~b}=\alpha_{2} e^{-\alpha_{1} \tau}, \mathrm{c}=\frac{\alpha_{3}}{\alpha_{1}}\|w\|_{\mathcal{L}_{\infty}}^{2} .
$$


Then the trivial solution of $(2.3)$ is finite-gain $\mathcal{L}_{\infty}$ stable from disturbance to output, and matrices $\mathrm{F}_{\mathrm{k}}, \mathrm{k}=1,2, \cdots$ are subject to

$$
\left[\begin{array}{cc}
\left(1-d_{k}\right) P+P F_{k}+F_{k}^{T} P & \left(S F_{k}\right)^{\top} \\
S F_{k} & -I
\end{array}\right]<0
$$

where

$\mathrm{P}=\mathrm{S}^{\mathrm{T}} \mathrm{S}, \mathrm{S}$ is the unique solution to Cholesky decomposition of the positive definite matrix $\mathrm{P}$.

Proof. Let $x(t)=x\left(t, t_{0}, \phi\right)$ be any solution of (2.3) with $x_{t_{0}}=\phi$, and $v(t, x)=x^{\top}(t) P x(t), t \in\left[t_{k-1}, t_{k}\right)$, $k \in N$. We shall show

$$
v(t) \leqslant\left(a-c+\frac{\alpha_{1} c}{\alpha_{1}+b}\right) e^{\left(\alpha_{1}+b\right)\left(t-t_{k-1}\right)}-\frac{\alpha_{1} c}{\alpha_{1}+b}, \quad t \in\left[t_{k-1}, t_{k}\right), \quad k \in N,
$$

where $a, b, c$ are defined in Theorem 3.1. We first show that

$$
v(t) \leqslant\left(a-c+\frac{\alpha_{1} c}{\alpha_{1}+b}\right) e^{\left(\alpha_{1}+b\right)\left(t-t_{0}\right)}-\frac{\alpha_{1} c}{\alpha_{1}+b}, \quad t \in\left[t_{0}, t_{1}\right)
$$

For $t \in\left[t_{0}, t_{1}\right)$, we have

$$
\begin{aligned}
D^{+} v(t)= & {\left[\begin{array}{c}
x(t) \\
x(t-\tau) \\
w(t)
\end{array}\right]^{\top}\left[\begin{array}{ccc}
A^{\top} P+P A-\alpha_{1} P & P B & P \\
B^{\top} P & -\alpha_{2} P & 0 \\
P & 0 & -\alpha_{3} I
\end{array}\right]\left[\begin{array}{c}
x(t) \\
x(t-\tau) \\
w(t)
\end{array}\right] } \\
& +\alpha_{1} v(t)+\alpha_{2} v(t-\tau)+\alpha_{3} w^{\top}(t) w(t)
\end{aligned}
$$

By condition (3.1), we get

$$
\mathrm{D}^{+} v(\mathrm{t}) \leqslant \alpha_{1} v(\mathrm{t})+\alpha_{2} v(\mathrm{t}-\tau)+\alpha_{3} w^{\top}(\mathrm{t}) w(\mathrm{t})
$$

Using the method of the variation of parameters, we get

$$
\begin{aligned}
v(t) \leqslant & v\left(t_{0}\right) e^{\alpha_{1}\left(t-t_{0}\right)}+\alpha_{2} \int_{t_{0}}^{t} e^{\alpha_{1}(t-s)} v(s-\tau) d s+\alpha_{3} \int_{t_{0}}^{t} e^{\alpha_{1}(t-s)}\|w(s)\|_{\mathcal{L}_{\infty}}^{2} d s \\
\leqslant & \lambda_{\max }(\mathrm{P})\|\varphi\|_{\tau}^{2} e^{\alpha_{1}\left(\mathrm{t}-\mathrm{t}_{0}\right)}+\alpha_{2} \int_{\mathrm{t}_{0}-\tau}^{\mathrm{t}-\tau} e^{\alpha_{1}(\mathrm{t}-\mathrm{s}-\tau)} v(s) \mathrm{d} s+\alpha_{3}\|w\|_{\mathcal{L}_{\infty}}^{2} \int_{\mathrm{t}_{0}}^{\mathrm{t}} e^{\alpha_{1}(\mathrm{t}-\mathrm{s})} \mathrm{d} s \\
\leqslant & \lambda_{\max }(\mathrm{P})\|\varphi\|_{\tau}^{2} e^{\alpha_{1}\left(\mathrm{t}-\mathrm{t}_{0}\right)}+\alpha_{2} \int_{\mathrm{t}_{0}-\tau}^{\mathrm{t}_{0}} e^{\alpha_{1}(\mathrm{t}-\mathrm{s}-\tau)} v(s) \mathrm{d} s+\alpha_{2} e^{-\alpha_{1} \tau} \int_{\mathrm{t}_{0}}^{\mathrm{t}} e^{\alpha_{1}(\mathrm{t}-\mathrm{s})} v(\mathrm{~s}) \mathrm{d} s \\
& +\frac{\alpha_{3}}{\alpha_{1}}\|w\|_{\mathcal{L}_{\infty}}^{2}\left(e^{\alpha_{1}\left(\mathrm{t}-\mathrm{t}_{0}\right)}-1\right) \\
\leqslant & \left.\lambda_{\max }(\mathrm{P})\|\phi\|_{\tau}^{2}\left(1+\frac{\alpha_{2}}{\alpha_{1}}-\frac{\alpha_{2}}{\alpha_{1}} e^{-\alpha_{1} \tau}\right)+\frac{\alpha_{3}}{\alpha_{1}}\|w\|_{\mathcal{L}_{\infty}}^{2}\right) e^{\alpha_{1}\left(\mathrm{t}-\mathrm{t}_{0}\right)}+\alpha_{2} e^{-\alpha_{1} \tau} \int_{\mathrm{t}_{0}}^{\mathrm{t}} e^{\alpha_{1}(\mathrm{t}-\mathrm{s})} v(\mathrm{~s}) \mathrm{d} s \\
& -\frac{\alpha_{3}}{\alpha_{1}}\|w\|_{\mathcal{L}_{\infty}}^{2} \\
= & \mathrm{a} e^{\alpha_{1}\left(\mathrm{t}-\mathrm{t}_{0}\right)}+\mathrm{b} \int_{\mathrm{t}_{0}}^{\mathrm{t}} e^{\alpha_{1}(\mathrm{t}-\mathrm{s})} v(\mathrm{~s}) \mathrm{d} s-\mathrm{c} .
\end{aligned}
$$

By Lemma 2.5,

$$
v(t) e^{-\alpha_{1} t} \leqslant a e^{-\alpha_{1} t_{0}}+b \int_{t_{0}}^{t} e^{-\alpha_{1} s} v(t) d s-c e^{-\alpha_{1} t}
$$




$$
\begin{aligned}
& \leqslant\left(a e^{-\alpha_{1} t_{0}}-c e^{-\alpha_{1} t_{0}}\right) e^{b\left(t-t_{0}\right)}+\int_{t_{0}}^{t} \alpha_{1} c e^{-\alpha_{1} s} e^{b(t-s)} d s \\
& =(a-c) e^{-\alpha_{1} t_{0}} e^{b\left(t-t_{0}\right)}+\alpha_{1} c \int_{t_{0}}^{t} e^{-\left(\alpha_{1}+b\right) s+b t} d s \\
& =(a-c) e^{-\alpha_{1} t_{0}} e^{b\left(t-t_{0}\right)}-\frac{\alpha_{1} c}{\alpha_{1}+b}\left(e^{-\alpha_{1} t}-e^{b t-\left(\alpha_{1}+b\right) t_{0}}\right) .
\end{aligned}
$$

Performing " $e^{\alpha_{1} t "}$ to both sides of the above inequality, we have

$$
\begin{aligned}
v(t) & \leqslant(a-c) e^{\left(\alpha_{1}+b\right)\left(t-t_{0}\right)}-\frac{\alpha_{1} c}{\alpha_{1}+b}\left(e^{-\alpha_{1} t}-e^{b t-\left(\alpha_{1}+b\right) t_{0}}\right) e^{\alpha_{1} t} \\
& =\left(a-c+\frac{\alpha_{1} c}{\alpha_{1}+b}\right) e^{\left(\alpha_{1}+b\right)\left(t-t_{0}\right)}-\frac{\alpha_{1} c}{\alpha_{1}+b} .
\end{aligned}
$$

Hence (3.7) holds and then (3.6) is true for $k=1$. By $a-c+\frac{\alpha_{1} c}{\alpha_{1}+b}>0$ and condition (3.3), we get

$$
v(t) \leqslant\left(a-c+\frac{\alpha_{1} c}{\alpha_{1}+b}\right) e^{\left(\alpha_{1}+b\right) \beta}-\frac{\alpha_{1} c}{\alpha_{1}+b}<\left(a-c+\frac{\alpha_{1} c}{\alpha_{1}+b}\right) e^{\left(\alpha_{1}+b\right) \beta} .
$$

Now we assume that (3.6) holds for $k=1,2, \cdots, m(m \in N)$, i.e.,

$$
v(t) \leqslant\left(a-c+\frac{\alpha_{1} c}{\alpha_{1}+b}\right) e^{\left(\alpha_{1}+b\right)\left(t-t_{k-1}\right)}-\frac{\alpha_{1} c}{\alpha_{1}+b}<\left(a-c+\frac{\alpha_{1} c}{\alpha_{1}+b}\right) e^{\left(\alpha_{1}+b\right)\left(t-t_{k-1}\right)}, t \in\left[t_{k-1}, t_{k}\right) .
$$

From conditions (3.2), (3.4) and (3.8), we have

$$
\begin{aligned}
v\left(t_{m}\right) & =x^{\top}\left(t_{m}^{-}\right)\left(I+F_{m}\right)^{\top} P\left(I+F_{m}\right) x\left(t_{m}^{-}\right) \leqslant d_{m} v\left(t_{m}^{-}\right) \\
& \leqslant \frac{(a-c)-\frac{\alpha_{2}}{\alpha_{1}}\left(a-c+\frac{\alpha_{1} c}{\alpha_{1}+b}\right) e^{\left(\alpha_{1}+b\right) \beta}\left(1-e^{-\alpha_{1} \tau}\right)}{\left(a-c+\frac{\alpha_{1} c}{\alpha_{1}+b}\right) e^{\left(\alpha_{1}+b\right)\left(t_{m}-t_{m-1}\right)}}\left(a-c+\frac{\alpha_{1} c}{\alpha_{1}+b}\right) e^{\left(\alpha_{1}+b\right)\left(t_{m}-t_{m-1}\right)} \\
& =(a-c)-\frac{\alpha_{2}}{\alpha_{1}}\left(a-c+\frac{\alpha_{1} c}{\alpha_{1}+b}\right) e^{\left(\alpha_{1}+b\right) \beta}\left(1-e^{-\alpha_{1} \tau}\right) .
\end{aligned}
$$

Next, we shall show that (3.6) holds for $k=m+1$, i.e.,

$$
v(t) \leqslant\left(a-c+\frac{\alpha_{1} c}{\alpha_{1}+b}\right) e^{\left(\alpha_{1}+b\right)\left(t-t_{m}\right)}-\frac{\alpha_{1} c}{\alpha_{1}+b}, t \in\left[t_{m}, t_{m+1}\right) .
$$

For $t \in\left[t_{m}, t_{m+1}\right)$, we always have

$$
\mathrm{D}^{+} v(\mathrm{t}) \leqslant \alpha_{1} v(\mathrm{t})+\alpha_{2} v(\mathrm{t}-\tau)+\alpha_{3}\|w\|_{\mathcal{L}_{\infty}}^{2} .
$$

Integrating from $t_{m}$ to $t$ gives

$$
v(t) \leqslant v\left(t_{m}^{+}\right) e^{\alpha_{1}\left(t-t_{m}\right)}+\alpha_{2} \int_{t_{m}}^{t} e^{\alpha_{1}(t-s)} v(s-\tau) d s+\alpha_{3} \int_{t_{m}}^{t} e^{\alpha_{1}(t-s)}\|w(s)\|_{\mathcal{L}_{\infty}}^{2} d s .
$$

By (3.9), we have

$$
\begin{aligned}
v(t) \leqslant & \left((a-c)-\frac{\alpha_{2}}{\alpha_{1}}\left(a-c+\frac{\alpha_{1} c}{\alpha_{1}+b}\right) e^{\left(\alpha_{1}+b\right) \beta}\left(1-e^{-\alpha_{1} \tau}\right)\right) e^{\alpha_{1}\left(t-t_{m}\right)} \\
& +\alpha_{2} \int_{t_{m}-\tau}^{t-\tau} e^{\alpha_{1}(t-s-\tau)} v(s) d s+\alpha_{3}\|w\|_{\mathcal{L}_{\infty}}^{2} \int_{t_{m}}^{t} e^{\alpha_{1}(t-s)} d s \\
\leqslant & \left((a-c)-\frac{\alpha_{2}}{\alpha_{1}}\left(a-c+\frac{\alpha_{1} c}{\alpha_{1}+b}\right) e^{\left(\alpha_{1}+b\right) \beta}\left(1-e^{-\alpha_{1} \tau}\right)\right) e^{\alpha_{1}\left(t-t_{m}\right)} \\
& +\alpha_{2} \int_{t_{\mathfrak{m}}-\tau}^{t_{m}} e^{\alpha_{1}(t-s-\tau)} v(s) d s+\alpha_{2} e^{-\alpha_{1} \tau} \int_{t_{m}}^{t} e^{\alpha_{1}(t-s)} v(s) d s
\end{aligned}
$$




$$
\begin{aligned}
& +\alpha_{3}\|w\|_{\mathcal{L}_{\infty}}^{2} \int_{\mathrm{t}_{\mathrm{m}}}^{\mathrm{t}} e^{\alpha_{1}(\mathrm{t}-\mathrm{s})} \mathrm{ds} \\
& \leqslant\left((a-c)-\frac{\alpha_{2}}{\alpha_{1}}\left(a-c+\frac{\alpha_{1} c}{\alpha_{1}+b}\right) e^{\left(\alpha_{1}+b\right) \beta}\left(1-e^{-\alpha_{1} \tau}\right)\right) e^{\alpha_{1}\left(t-t_{m}\right)} \\
& +\alpha_{2} \int_{t_{m}-\tau}^{t_{m}} e^{\alpha_{1}(t-s-\tau)}\left(\left(a-c+\frac{\alpha_{1} c}{\alpha_{1}+b}\right) e^{\left(\alpha_{1}+b\right) \beta}\right) d s \\
& +\alpha_{2} e^{-\alpha_{1} \tau} \int_{t_{m}}^{t} e^{\alpha_{1}(t-s)} v(s) d s+\frac{\alpha_{3}}{\alpha_{1}}\|w\|_{\mathcal{L}_{\infty}}^{2} e^{\alpha_{1}\left(t-t_{m}\right)}-\frac{\alpha_{3}}{\alpha_{1}}\|w\|_{\mathcal{L}_{\infty}}^{2} \\
& \leqslant\left((a-c)-\frac{\alpha_{2}}{\alpha_{1}}\left(a-c+\frac{\alpha_{1} c}{\alpha_{1}+b}\right) e^{\left(\alpha_{1}+b\right) \beta}\left(1-e^{-\alpha_{1} \tau}\right)\right) e^{\alpha_{1}\left(t-t_{m}\right)} \\
& +\frac{\alpha_{2}}{\alpha_{1}}\left(1-e^{-\alpha_{1} \tau}\right) e^{\left(\alpha_{1}+b\right) \beta}\left(a-c+\frac{\alpha_{1} c}{\alpha_{1}+b}\right) e^{\alpha_{1}\left(t-t_{m}\right)}+\frac{\alpha_{3}}{\alpha_{1}}\|w\|_{\mathcal{L}_{\infty}}^{2} e^{\alpha_{1}\left(t-t_{m}\right)} \\
& +\alpha_{2} e^{-\alpha_{1} \tau} \int_{t_{m}}^{t} e^{\alpha_{1}(t-s)} v(s) d s-\frac{\alpha_{3}}{\alpha_{1}}\|w\|_{\mathcal{L}_{\infty}}^{2} \\
& \leqslant a e^{\alpha_{1}\left(t-t_{m}\right)}+b \int_{t_{m}}^{t} e^{\alpha_{1}(t-s)} v(s) d s-c .
\end{aligned}
$$

Applying Lemma 2.5 again gives

$$
v(t) \leqslant\left(a-c+\frac{\alpha_{1} c}{\alpha_{1}+b}\right) e^{\left(\alpha_{1}+b\right)\left(t-t_{m}\right)}-\frac{\alpha_{1} c}{\alpha_{1}+b}<\left(a-c+\frac{\alpha_{1} c}{\alpha_{1}+b}\right) e^{\left(\alpha_{1}+b\right)\left(t-t_{m}\right)} .
$$

Hence (3.6) holds for $k=m+1$. Thus by mathematical induction, we obtain that (3.6) holds, and hence we have

$$
v(t) \leqslant\left(a-c+\frac{\alpha_{1} c}{\alpha_{1}+b}\right) e^{\left(\alpha_{1}+b\right) \beta}-\frac{\alpha_{1} c}{\alpha_{1}+b} \leqslant\left(a-c+\frac{\alpha_{1} c}{\alpha_{1}+b}\right) e^{\left(\alpha_{1}+b\right) \beta}, \quad t \in\left[t_{k-1}, t_{k}\right) .
$$

From $\lambda_{\min }(P)\|x(t)\|^{2} \leqslant v(t) \leqslant a+\frac{2 \alpha_{1}-b}{\alpha_{1}-b} c$, it is clear that for $t \in\left[t_{k-1}, t_{k}\right)$,

$$
\begin{aligned}
\|x(t)\| & \leqslant \sqrt{\frac{\lambda_{\max }(\mathrm{P})\|\phi\|_{\tau}^{2}\left(\alpha_{1}+\alpha_{2}-\alpha_{2} e^{-\alpha_{1} \tau}\right)}{\alpha_{1} \lambda_{\min }(\mathrm{P})}+\frac{\alpha_{1} c}{\lambda_{\min }(\mathrm{P})\left(\alpha_{1}+\mathrm{b}\right)}} \\
& \leqslant \sqrt{\frac{\lambda_{\max }(\mathrm{P})\|\phi\|_{\tau}^{2}\left(\alpha_{1}+\alpha_{2}-\alpha_{2} e^{-\alpha_{1} \tau}\right)}{\alpha_{1} \lambda_{\min }(\mathrm{P})}}+\sqrt{\frac{\alpha_{1} \alpha_{3}}{\lambda_{\min }(\mathrm{P}) \alpha_{1}\left(\alpha_{1}+\alpha_{2} e^{-\alpha_{1} \tau}\right)}}\|w\|_{\mathcal{L}_{\infty} .} .
\end{aligned}
$$

So,

$$
\begin{aligned}
\|y\| & \leqslant\|C\|\|x(t)\| \leqslant\|C\| \sqrt{\frac{\lambda_{\max }(\mathrm{P})\|\phi\|_{\tau}^{2}\left(\alpha_{1}+\alpha_{2}-\alpha_{2} e^{-\alpha_{1} \tau}\right)}{\alpha_{1} \lambda_{\min }(\mathrm{P})}}+\|C\| \sqrt{\frac{\alpha_{1} \alpha_{3}}{\lambda_{\min }(\mathrm{P}) \alpha_{1}\left(\alpha_{1}+\alpha_{2} e^{-\alpha_{1} \tau}\right)}}\|w\|_{\mathcal{L}_{\infty}} \\
& =\gamma\|w\|_{\mathcal{L}_{\infty}}+\theta
\end{aligned}
$$

where

$$
\begin{aligned}
& \gamma=\|C\| \sqrt{\frac{\alpha_{1} \alpha_{3}}{\lambda_{\min }(\mathrm{P}) \alpha_{1}\left(\alpha_{1}+\alpha_{2} \mathrm{e}^{-\alpha_{1} \tau}\right)}}, \\
& \theta=\|\mathrm{C}\| \sqrt{\frac{\lambda_{\max }(\mathrm{P})\|\phi\|_{\tau}^{2}\left(\alpha_{1}+\alpha_{2}-\alpha_{2} \mathrm{e}^{-\alpha_{1} \tau}\right)}{\alpha_{1} \lambda_{\min }(\mathrm{P})}} .
\end{aligned}
$$

By Definition 2.2, the trivial solution of systems (2.3) is finite-gain $\mathcal{L}_{\infty}$ stability from disturbance to output and due to condition (3.2) and Schur complement, matrices $F_{k}, k=1,2, \cdots$ are subject to condition (3.5) in Theorem 3.1.

Remark 3.2. In LMI (3.1) of Theorem 3.1, the constant $\alpha_{1}>0$ allows the matrix $A$ is unstable, which measures the degree of instability of the delay-free systems and is determined by the eigenvalues of the 
matrix $A$, while $\alpha_{2}$ and $\alpha_{3}$ are determined by the matrix B. The constants $d_{k}$ in conditions (3.2) and (3.4) measure the amplitude of control impulses. Inequality (3.3) characterized the relationship among the interval length of consecutive impulses and the other parameters. As we can see from Theorem 3.1, impulses have played an important role in finite-gain $\mathcal{L}_{\infty}$ stabilizing the delayed differential systems. Moreover, due to the positive-definite property of matrix $\mathrm{P}$, we can get a unique Cholesky decomposition of the form $P=S^{\top} S$, where $S$ is the upper triangular matrix. Therefore, nonlinear condition (3.2) can be equivalent to LMIs (3.5) by Schur complement easily. When $A$ is stable, the finite-gain $\mathcal{L}_{\infty}$ stability results of systems (2.3) are given as follows, where impulses are treated as disturbances.

Theorem 3.3. Assume that there exist a positive definite matrix $\mathrm{P}$ and constants $\alpha_{i} \in \mathrm{R}^{+}, \mathrm{i}=1,2,3, \mathrm{~d}_{\mathrm{k}} \in$ $\mathrm{R}^{+}, \mathrm{k} \in \mathrm{N}$, such that $\alpha_{1}>\alpha_{2} e^{\alpha_{1} \tau}$, and

$$
\begin{aligned}
& {\left[\begin{array}{ccc}
A^{\top} P+P A+\alpha_{1} P & P B & P \\
* & -\alpha_{2} P & 0 \\
* & * & -\alpha_{3} I
\end{array}\right] \leqslant 0,} \\
& \left(I+F_{k}\right)^{\top} P\left(I+F_{k}\right)-d_{k} P \leqslant 0 \text { for each } k=1,2, \cdots,
\end{aligned}
$$

and

$$
\tau \leqslant t_{k}-t_{k-1}, 1<d_{k} \leqslant \frac{a-\frac{\alpha_{2}}{\alpha_{1}}\left(a+\frac{2 \alpha_{1}-b}{\alpha_{1}-b} c\right)\left(e^{\alpha_{1} \tau}-1\right)+c}{(a+c) e^{-\left(\alpha_{1}-b\right)\left(t_{k}-t_{k-1}\right)}+\frac{\alpha_{1} c}{\alpha_{1}-b}},
$$

where

$$
a=\lambda_{\max }(P)\|\phi\|_{\tau}^{2}+\frac{\alpha_{2}}{\alpha_{1}} \lambda_{\max }(\mathrm{P})\|\phi\|_{\tau}^{2}\left(e^{\alpha_{1} \tau}-1\right)-\frac{\alpha_{3}}{\alpha_{1}}\|w\|_{\mathcal{L}_{\infty}}^{2}, \mathrm{~b}=\alpha_{2} e^{\alpha_{1} \tau}, \mathrm{c}=\frac{\alpha_{3}}{\alpha_{1}}\|w\|_{\mathcal{L}_{\infty}}^{2} .
$$

Then the trivial solution of (2.3) is finite-gain $\mathcal{L}_{\infty}$ stable from disturbance to output, and matrices $F_{k}, k=1,2, \cdots$, are subject to

$$
\left[\begin{array}{cc}
\left(1-d_{k}\right) P+P F_{k}+F_{k}^{T} P & \left(S F_{k}\right)^{\top} \\
S F_{k} & -I
\end{array}\right]<0,
$$

where

$$
\mathrm{P}=\mathrm{S}^{\top} \mathrm{S}, \quad \mathrm{S} \text { is the unique solution to Cholesky decomposition of the positive definite matrix } \mathrm{P} .
$$

Proof. Let $x(t)=x\left(t, t_{0}, \phi\right)$ be any solution of systems (2.3) with $x_{t_{0}}=\phi$, and $v(t)=x^{\top}(t) P x(t), t \in$ $\left[t_{k-1}, t_{k}\right), k \in N$. Similar to the proof of Theorem 3.1, we could get

$$
v(t) \leqslant(a+c) e^{-\left(\alpha_{1}-b\right)\left(t-t_{k-1}\right)}+\frac{\alpha_{1} c}{\alpha_{1}-b} \leqslant a+\frac{2 \alpha_{1}-b}{\alpha_{1}-b} c, \quad t \in\left[t_{k-1}, t_{k}\right), \quad k \in N .
$$

From $\lambda_{\min }(P)\|x(t)\|^{2} \leqslant v(t) \leqslant a+\frac{2 \alpha_{1}-b}{\alpha_{1}-b} c$, it is clear that for $t \in\left[t_{k-1}, t_{k}\right)$,

$$
\begin{aligned}
\|x(t)\| & \leqslant \sqrt{\frac{\lambda_{\max }(\mathrm{P})\|\phi\|_{\tau}^{2}+\frac{\alpha_{2}}{\alpha_{1}} \lambda_{\max }(\mathrm{P})\|\phi\|_{\tau}^{2}\left(e^{\alpha_{1} \tau}-1\right)+\frac{\alpha_{1}}{\alpha_{1}-\mathrm{b}} \mathrm{c}}{\lambda_{\min }(\mathrm{P})}} \\
& \leqslant \sqrt{\frac{\lambda_{\max }(\mathrm{P})\|\phi\|_{\tau}^{2}+\frac{\alpha_{2}}{\alpha_{1}} \lambda_{\max }(\mathrm{P})\|\phi\|_{\tau}^{2}\left(e^{\alpha_{1} \tau}-1\right)}{\lambda_{\min }(\mathrm{P})}}+\sqrt{\frac{\alpha_{1} c}{\lambda_{\min }(\mathrm{P})\left(\alpha_{1}-b\right)}} .
\end{aligned}
$$

So,

$$
\begin{aligned}
\|y\| & \leqslant\|C\|\|x(t)\| \leqslant\|C\| \sqrt{\frac{\lambda_{\max }(\mathrm{P})\|\phi\|_{\tau}^{2}+\frac{\alpha_{2}}{\alpha_{1}} \lambda_{\max }(\mathrm{P})\|\phi\|_{\tau}^{2}\left(\mathrm{e}^{\alpha_{1} \tau}-1\right)}{\lambda_{\min }(\mathrm{P})}}+\|C\| \sqrt{\frac{\alpha_{3}}{\lambda_{\min }(\mathrm{P})\left(\alpha_{1}-\mathrm{b}\right)}}\|w\|_{\mathcal{L}_{\infty}}, \\
& =\gamma\|w\|_{\mathcal{L}_{\infty}}+\theta
\end{aligned}
$$

where

$$
\begin{aligned}
& \gamma=\|C\| \sqrt{\frac{\alpha_{3}}{\lambda_{\min }(\mathrm{P})\left(\alpha_{1}-\mathrm{b}\right)}}, \\
& \theta=\|C\| \sqrt{\frac{\alpha_{1} \lambda_{\max }(\mathrm{P})\|\phi\|_{\tau}^{2}+\alpha_{2} \lambda_{\max }(\mathrm{P})\|\phi\|_{\tau}^{2}\left(e^{\alpha_{1} \tau}-1\right)}{\alpha_{1} \lambda_{\min }(\mathrm{P})}} .
\end{aligned}
$$

By Definition 2.2, the trivial solution of systems (2.3) is finite-gain $\mathcal{L}_{\infty}$ stable from disturbance to output 
and the feedback gain matrices are subject to LMIs (3.13) in Theorem 3.3.

Remark 3.4. In LMI (3.10) of Theorem 3.3, the constant $\alpha_{1}>0$ requires $A$ is stable. Therefore, impulses in systems (2.3) are regarded as disturbances. Constants $\alpha_{i}, d_{k}>0$ in (3.11) and (3.12) determine and measure the amplitude of impulsive disturbances. The analysis on matrices $F_{k}, k=1,2, \cdots$ in Theorem 3.3 are same to that in Remark 3.2.

\section{Examples}

This section gives two examples to demonstrate the effectiveness of the proposed approach.

Example 4.1. Consider the systems with

$$
A=\left[\begin{array}{ll}
1.5 & 1.9 \\
2.3 & 0.5
\end{array}\right], \quad B=\left[\begin{array}{cc}
-0.3 & 0.2 \\
0.4 & -0.3
\end{array}\right], \quad C=\left[\begin{array}{ll}
2 & 1 \\
1 & 0
\end{array}\right]
$$

Solving LMI (3.1) gives us a positive definite matrix $P=\left[\begin{array}{ll}0.1720 & 0.0667 \\ 0.0667 & 0.1354\end{array}\right]$ with $\alpha_{1}=12.3319, \alpha_{2}=$ 1.8391, $\alpha_{3}=0.2400$ and $\lambda_{\min }(\mathrm{P})=0.0845, \lambda_{\max }(\mathrm{P})=0.2228$. For $\varphi=\left[\begin{array}{l}3 \\ 4\end{array}\right], w(t)=\left[\begin{array}{c}0 \\ 5 \cos t\end{array}\right]$, we have $a=6.1529, b=1.6257, c=0.4865$. Therefore, $0.01=\tau \leqslant t_{m}-t_{m-1} \leqslant \beta \leqslant 0.2854, m=1,2, \cdots$. Figure 1 shows the trajectory of output $y(t)$ without the impulsive control. Figure 2 shows the bound of output $y(t)$ for $t_{m}-t_{m-1}=0.04, m=1,2, \cdots, \beta=0.0837, d_{m}=0.5000$ with the stabilizing feedback gain matrix $F=\left[\begin{array}{cc}-0.8418 & 0.0620 \\ 0.0621 & -0.8756\end{array}\right]$. From the results, we can get impulses play a crucial role on finite-gain $\mathcal{L}_{\infty}$ stability from disturbance to output of dynamic systems.

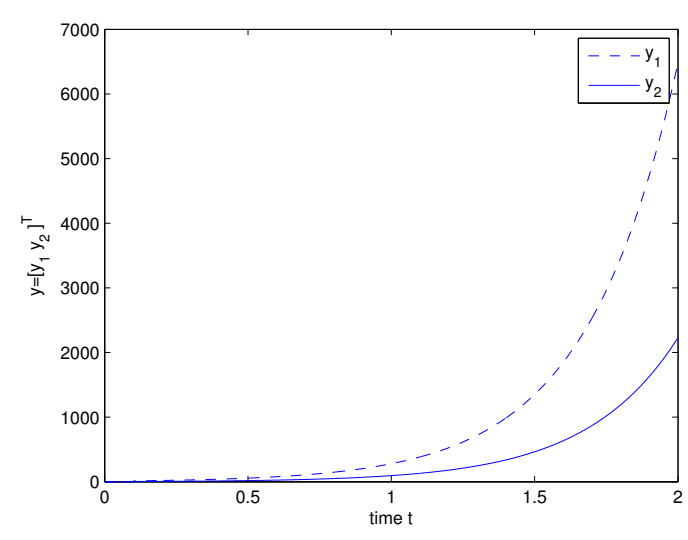

Figure 1: output without impulsive control in (4.1).

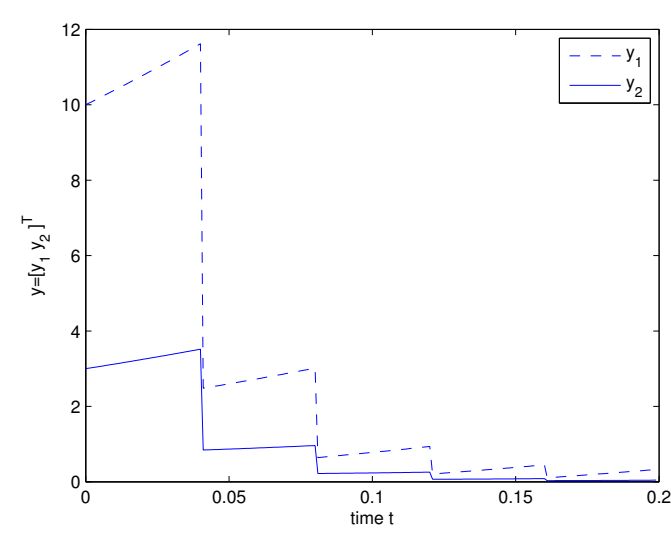

Figure 2: output with impulsive control in (4.1).

Remark 4.2. The trivial solution of the corresponding delayed equations (4.1) without impulses is unstable (see Figure 1), as we can see in Figure 2, impulses do contribute to the finite-gain $\mathcal{L}_{\infty}$ stabilization from disturbance to output of the systems.

Example 4.3. Consider the systems (2.3) with

$$
A=\left[\begin{array}{cc}
-2 & 1 \\
1 & -2
\end{array}\right], \quad B=\left[\begin{array}{cc}
-0.4 & 0 \\
0 & -1
\end{array}\right], \quad C=\left[\begin{array}{ll}
2 & 1 \\
1 & 0
\end{array}\right]
$$

Solving LMI (3.10) gives us a positive definite matrix $P=\left[\begin{array}{cc}0.3625 & -0.2338 \\ -0.2338 & 0.1922\end{array}\right]$ with $\alpha_{1}=1.0676, \alpha_{2}=$ 
0.6538, $\alpha_{3}=0.4942$, and $\lambda_{\min }(\mathrm{P})=0.0285, \lambda_{\max }(\mathrm{P})=0.5261$. For $\varphi=\left[\begin{array}{c}3 \\ -4\end{array}\right], w(\mathrm{t})=\left[\begin{array}{c}0 \\ \text { cost }\end{array}\right]$, we get $a=13.5971, b=0.7275<\alpha_{1}, c=0.4629,0<d_{m} \leqslant 3.2315, m=1,2, \cdots$ and the stabilizing feedback gain matrix $F=\left[\begin{array}{ll}0.4619 & 0.0525 \\ 0.0525 & 0.5001\end{array}\right]$. Figure 3 shows the bound of output $y(t)$ without disturbance, while Figure 4 shows the bound of output $y(t)$ with the impulsive disturbance, where $0.1=\tau \leqslant t_{m}-t_{m-1}=$ $5, \mathrm{~d}_{\mathrm{m}}=3.2315, \mathrm{~m}=1,2, \cdots$.

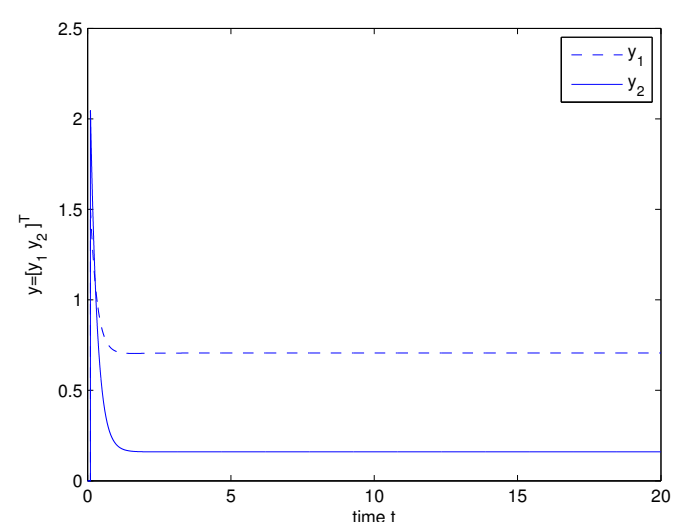

Figure 3: output without impulsive control in (4.2).

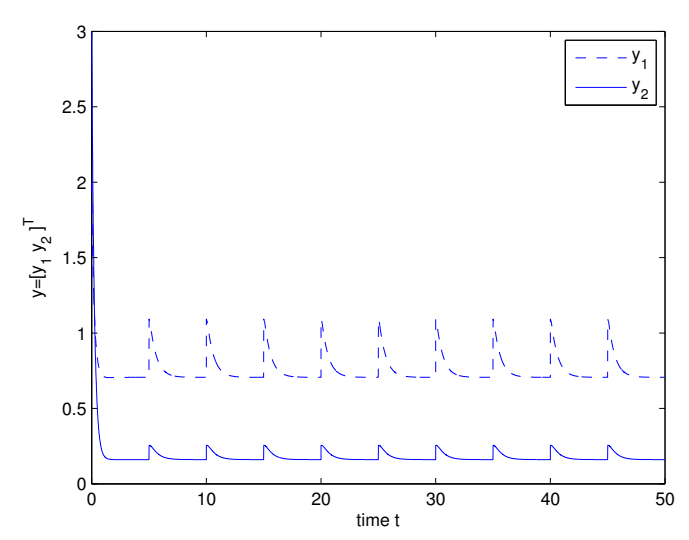

Figure 4: output with impulsive control in (4.2).

Remark 4.4. Figure 3 and Figure 4 show that the stable systems (4.2) can be made finite-gain $\mathcal{L}_{\infty}$ stable from disturbance to output under an appropriate sequence of impulses treated as disturbances.

Remark 4.5. The finite-gain $\mathcal{L}_{\infty}$ stability refers to any bounded exogenous inputs (here $w$ ). If the exogenous input is regarded as the disturbances, for example, the smaller the better for the bound of output. On the other hand, the larger the better for the same systems if it is considered as the amplifier. Therefore, we just take two examples to illustrate the efficiency of the proposed method without any comparisons. In our future work, we will adopt delay decomposition approach to vary the bounds of output by tuning the parameters.

\section{Conclusions}

In this paper, we have studied the disturbance-output property of delayed systems via impulsive control. Encouragingly, this method may be potentially applied to the exponential stability of impulsive systems or impulsive systems with the time-varying delay. This will be our research in future. Our contributions are as follows:

(1) Compared with the existing results on the analysis of input-output stability, our criteria are established by the method of Lyapunov and LMI tools instead of small gain theory or transfer function.

(2) Our results reveal that the impulse has great impacts on the model. Using impulsive feedback control, the linear delayed differential systems can be finite-gain $\mathcal{L}_{\infty}$ stabilized from $w$ to $y$ even if the delay-free systems are unstable. On the other hand, delay differential equations also may be guaranteed finitegain $\mathcal{L}_{\infty}$ stable from $w$ to $y$ when impulses are treated as disturbances. The effects of the impulses are considered in detail.

\section{Acknowledgment}

This work was supported by the Fundamental Research Funds for the Central Universities, Southwest University for Nationalities (2017NZYQN12), Innovative Research Teams of the Education Department of 
Sichuan Province (15TD0050) and the National Natural Science Foundation of China (61273007), and the Natural Sciences and Engineering Research Council of Canada (NSERC).

\section{References}

[1] K. Akbari Moornani, M. Haeri, Necessary and sufficient conditions for BIBO-stability of some fractional delay systems of neutral type, IEEE Trans. Automat. Control, 56 (2011), 125-128. 1

[2] F. Amato, G. De Tommasi, A. Pironti, Necessary and sufficient conditions for finite-time stability of impulsive dynamical linear systems, Automatica J. IFAC, 49 (2013), 2546-2550. 1

[3] C. Bonnet, J. R. Partington, Analysis of fractional delay systems of retarded and neutral type, Automatica J. IFAC, 38 (2002), 1133-1138. 1

[4] J. Carvajal, G.-R. Chen, H. Ogmen, Fuzzy PID controller: design, performance evaluation, and stability analysis, Analytical theory of fuzzy control with applications, Inform. Sci., 123 (2000), 249-270. 1

[5] Y. Ji, X.-M. Liu, Unified synchronization criteria for hybrid switching-impulsive dynamical networks, Circuits Systems Signal Process., 34 (2015),1499-1517. 1

[6] H. K. Khalil, Nonlinear systems, Third edition, Prentice-Hall, Upper Saddle River, NJ, (2002). 2.3

[7] P. Li, S.-M. Zhong, BIBO stabilization of time-delayed system with nonlinear perturbation, Appl. Math. Comput., 195 (2008), 264-269. 1

[8] P. Li, S.-M. Zhong, J.-Z.Cui, Delay-dependent robust BIBO stabilization of uncertain system via LMI approach, Chaos Solitons Fractals, 40 (2009), 1021-1028. 1

[9] J.-Q. Lu, D. W. C. Ho, J.-D. Cao, A unified synchronization criterion for impulsive dynamical networks, Automatica J. IFAC, 46 (2010), 1215-1221. 1

[10] A. Möller, U. T. Jönsson, Input-output analysis of power control in wireless networks, IEEE Trans. Automat. Control, 58 (2013), 834-846. 1

[11] P. Naghshtabrizi, J. P. Hespanha, A. R. Teel, Stability of delay impulsive systems with application to networked control systems, Trans. Inst. Measurement Control, 32 (2010), 511-528. 1

[12] J. R. Partington, C. Bonnet, $\mathrm{H}_{\infty}$ and BIBO stabilization of delay systems of neutral type, Syst. Control Lett., 52 (2004), 283-288. 1

[13] K.-B. Shi, Y.-Y. Tang, X.-Z. Liu, S.-M. Zhong, Non-fragile sampled-data robust synchronization of uncertain delayed chaotic Lurie systems with randomly occurring controller gain fluctuation, ISA Trans., 66 (2017), 185-199.

[14] A. R. Teel, T. T. Georgiou, L. Praly, E. D. Sontag, Input-output stability, W.S. Levine (Ed.), The Control Handbook, CRC Press, Boca Raton, FL, (1996), 895-908. 1

[15] M. Turinici, Abstract comparison principles and multivariable Gronwall-Bellman inequalities, J. Math. Anal. Appl., 117 (1986), 100-127. 2.5

[16] W.-A. Zhang, L. Yu, A robust control approach to stabilization of networked control systems with time-varying delays, Automatica J. IFAC, 45 (2009), 2440-2445. 1

[17] L. Zhao, H.-J. Gao, H. R. Karimi, Robust stability and stabilization of uncertain T-S fuzzy systems with time-varying delay: an input-output approach, IEEE Trans. Fuzzy Syst., 21 (2013), 883-897. 1 